\title{
MLE activates transcription via the minimal transactivation domain in Drosophila
}

\author{
SATOKO ARATANI ${ }^{1}$, YUJI KAGEYAMA ${ }^{2}$, AKIRA NAKAMURA ${ }^{3}$, HIDETOSHI FUJITA ${ }^{1}$, \\ RYOJI FUJII ${ }^{1}$, KUSUKI NISHIOKA ${ }^{4}$ and TOSHIHIRO NAKAJIMA ${ }^{1}$
}

\begin{abstract}
${ }^{1}$ Department of Locomotor Science, Institute of Medical Science, St. Marianna University School of Medicine, Kawasaki, Kanagawa 216-8512; ${ }^{2}$ Precursory Research for Embryonic Science and Technology, Japan Science and Technology Agency, Saitama 332-0012; ${ }^{3}$ Laboratory for Germline Development, RIKEN Center for Developmental Biology, Kobe, Hyogo 650-0047; ${ }^{4}$ Rheumatology, Immunology and Genetics, Program, Institute of Medical Science, St. Marianna University School of Medicine, Kawasaki, Kanagawa 216-8512, Japan
\end{abstract}

Received November 29, 2007; Accepted January 9, 2008

\begin{abstract}
Transcription levels of the genes on X chromosome are regulated through the dosage compensation mechanisms. The dosage compensation complex (DCC) localizes to $\mathrm{X}$ chromosome and activates the transcription of target genes in male 2-fold more than in female. Drosophila maleless (MLE), an ATPase/helicase, is a component of the DCC and essential for the viability of male flies. However, the functions of MLE on gene expression are not clear. RNA helicase A (RHA) is a homologue of Drosophila MLE and mediates the expression of several genes. RHA recruits preinitiation complex via the minimal transactivation domain (MTAD), consisting of 50 amino acids to target promoters. The tryptophan residues in MTAD are important for transactivation via RHA. The amino acid sequence of MTAD is conserved in MLE. In this study, we assessed whether the functions of MTAD are conserved in fruit fly by investigating the transcriptional activity of MLE. Transactivation assay indicated the MTAD of MLE had transcriptional activity in Schneider's cells. In vitro binding assays revealed that MLE recruited RNA polymerase II (Pol II) complexes through MTAD. Reporter assays showed that the MTAD, especially tryptophan residues, is important for transcription from roX promoter, similar to RHA. The results confirm that MTAD of MLE mediates the expression of MLE target genes through recruitment of Pol II.
\end{abstract}

\section{Introduction}

Drosophila males have a single $\mathrm{X}$ chromosome, while females have two $X$ chromosomes. In male flies, the dosage

Correspondence to: Dr Toshihiro Nakajima, Department of Genome Science, Institute of Medical Science, St. Marianna University School of Medicine, 2-16-1 Sugao, Miyamae-ku, Kawasaki, Kanagawa 216-8512, Japan

E-mail: nakashit@marianna-u.ac.jp

Key words: Drosophila, X chromosome, ATPase/helicase compensation mechanisms elevate the level of transcription derived from a single $\mathrm{X}$ chromosome to a level equivalent to that derived from the two $\mathrm{X}$ chromosome in the female (1). To date, five proteins; the dosage compensation complex (DCC), male-specific lethal 1 (MSL-1) $(2,3)$, male-specific lethal 2 (MSL-2) (4), male-specific lethal 3 (MSL-3) (5), male absent on the first (MOF) (6) and maleless (MLE) (7), have been identified by genetic analysis. Only the expression of MSL-2 is limited to Drosophila male (8). Loss-of function of these proteins results in male-specific lethality (9). In addition to these proteins, two noncoding RNAs; RNA on the X1 (roX1) and RNA on the X2 (roX2), which are the target genes of the DCC on the X chromosome, are also involved in this complex (10-12). It is well known that lysine 16 of histone $\mathrm{H} 4$ (H4K16) on X chromosome is highly acetylated in male flies $(13,14)$. The DCC colocalizes with the acetylated lysines.

The dosage compensation mechanism is divided into two steps $(15,16)$. In the first step, MSL-1 and MSL-2 form a core complex. This immature complex binds to 35 chromatin entry sites on the $\mathrm{X}$ chromosome and activates the transcription of target genes, including roX1 and roX2 genes $(17,18)$. In the second step, other components, such as MOF, MSL-3, roX RNAs and MLE, are recruited into the core complex to form the mature DCC. Subsequently, the DCC spreads onto the X chromosome and targets hundreds of the specific sites. MSL-1, MSL-2 and MSL3 are thought to have structural and scaffold roles (19), whereas MOF is a histone acetyltransferase, a member of the MYST family, and acetylates histone H4 on lysine $16(6,20)$. The noncoding RNAs, roX1 and roX2 genes, have little sequence homology to each other and the DNaseI hypersensitive site (DHS) in the genome. It is thought that the DCC are recruited to the DHS and activate the expression of the genes $(18,21,22)$. These RNAs contribute to the correct localization of the DCC on X chromosome (23). MSL-2, MSL-3 and MOF bind with MSL-1 via distinct domain (24), while MLE, which can bind nucleic acids (33), interacts with the complex through roX RNA, its recruitment of MLE on the $\mathrm{X}$ chromosome is RNase-sensitive (25). MLE is an ATPase/ helicase and an essential factor for the development of fruit fly (26). Spontaneous MLE null mutant ( $\left.\mathrm{mle}^{1}\right)$ has been identified 
and the homozygous mle ${ }^{1}$ male die during the larval stage (27). The enzymatic activity of this protein is necessary for the dosage compensation $(25,26)$.

Mechanisms, which are different from Drosophila, regulate the products of gene expression from $\mathrm{X}$ chromosome in mammalian cells. The mammalian cells have genes coding the components of the DCC except for roX RNAs $(28,29)$. Human MSL proteins are reported to work as a histone acetyltransferase complex, however the details are still unknown (30). While the functions of MLE in Drosophila dosage compensation remain to be elucidated, many functions of RNA helicase A (RHA), a mammalian homologue of MLE (31), on gene expression were delineated by our group (32-34).

RHA belongs to the DExH helicase/ATPase family and unwinds both of double-stranded RNA and DNA from 3 ' to 5'. RHA has several functional domains (35-37). There are two double-stranded RNA binding domains, dsRBDI and dsRBDII in the amino terminus of RHA. The catalytic core domain contains well-conserved motifs and the ATP binding site, and is located in the central region of RHA (38). The minimal transactivation domain (MTAD) is next to the ATP binding site (39). The carboxyl terminus region contains glycine-rich single-stranded nucleic acid binding domain (RGG) and the nuclear localization signal (NLS) $(40,41)$. RHA and MLE have $50 \%$ sequence identity and $90 \%$ similarity to each other (31).

RHA has various functions at several stages of gene expression in mammalian cells. We reported previously that RHA interacts with the cAMP responsive element binding protein (CREB) binding protein (CBP) and mediates the recruitment of RNA polymerase II (Pol II) complexes through MTAD to promoters $(34,39)$. RHA also regulates several transactivations, such as BRCA1 (42), the activation function domain of mineralocorticoid receptor (43). RHA activates transcription by binding to nucleic acids, such as the cis-acting transactivation response element (TAR) of HIV-1 (33) and the p16INK4a promoter (44).

Several studies have emphasized the importance of RHA. The present study was designated to establish transcription and to verify that MTAD of MLE acts as transactivation domain in fly cells such as RHA in mammalian cells. We indicated that MTAD was included in the recruitment of Pol II to the DCC and regulated transcription from X chromosome, and that MLE acts as a transcriptional regulator not as a complex.

\section{Materials and methods}

Plasmids. To construct the mle transgene, a SpeI/XbaI fragment that included the mle gene and the franking region (7) was isolated from Drosophila genomic library using the $\mathrm{C}$ terminal region of mle (Research Genetics) as a probe. This $10.2 \mathrm{~kb}$ fragment was filled in pCaSpeR4. For the deletion mutant transgene, the $\triangle \mathrm{MTAD}$ fragment was generated by PCR and substituted for wild-type transgene. The expression vector in Drosophila cells, pBlue-Actin5C, in cells was constructed by inserting Actin5C promoter derived from pWA GAL4 into pBluescript II. The full length cDNAs coding MLE and the deletion mutant including MTAD (amino acids 219-653), termed MLE2, were generated by PCR and subcloned into pBlue-Actin5C. Amino acids 326378 were deleted from MLE2 and full length MLE to generate MLE2 $\triangle \mathrm{MTAD}$ and MLE $\triangle \mathrm{MTAD}$, respectively. The mutants with substitutions of amino acids were also made by PCR. For glutathione S-transferase pull down assay the full-length and MLE2 fragments were inserted into the bacterial expression vector pGEX-6P-1 (Amersham). There tryptophan residues in the MTAD region were substituted with alanine, phenylalanine or leucine and these mutants were termed MTADW328A, MTADW328F, MTADW328L, MTADW335A, MTADW335F, MTADW335L, MTADW338A, MTADW338F and MTADW338L. Each of these fragments was inserted and fused to the GAL4 DNA binding domain (GAL4-DBD), into pBlue-Actin5C or pcDNA3 (Invitrogen), for transactivation assays in Drosophila or mammalian cells, respectively. The plasmids encoding the enhanced green fluorescent protein fusion protein (GFPMLE) were constructed by inserting fragments of the MLE wild-type and each mutants into pEGFP-C2 (Clontech Laboratories). The C-terminal deletion mutant (MLE $\triangle \mathrm{EN})$, from which was deleted the amino acid region 1163 to 1287 , was generated by PCR and inserted into pEGFP-C2. For reporter assay, the G14-ts/BasicVector 2 was constructed by subcloning 14 UAS fragments and transposase promoterderived from pUASp into BasicVector2 (Toyo ink). Four DHS fragments and roX2 promoter generated by PCR was constructed in BasicVector2 (45). The heat shock promoter derived from pUAST was inserted into BasicVector2 and a reporter plasmid was generated (hs/BasicVector2). As an internal control vector, $B$-galactosidase cDNA was inserted into pBlue-Actin5C.

GST pull down assay. The GST fusion proteins of each MLE2 mutant and the full length MLE were expressed in Escherichia coli strain TopXF' (Invitrogen) and purified using glutathione sepharose beads (Amersham Pharmacia Biotech). Then, $100 \mu \mathrm{g}$ of nuclear extracts obtained from Shneider's cells (SL-2) were incubated with $2 \mu \mathrm{g}$ of each GST fusion protein bound to resin in $1 \mathrm{ml}$ of buffer A [50 mM HEPES (pH 7.9), $150 \mathrm{mM} \mathrm{NaCl}, 1 \mathrm{mM}$ dithiothreitol (DTT), $1 \mathrm{mM}$ EDTA, $1 \mathrm{mM} \mathrm{MgCl} 2,0.05 \%$ NP-40, $1 \mathrm{mM} \mathrm{Na}_{3} \mathrm{VO}_{4}, 5 \mathrm{mM}$ $\mathrm{NaF}, 1 \mu \mathrm{g} / \mathrm{ml}$ aprotinin, $1 \mu \mathrm{g} / \mathrm{ml}$ leupeptin, and $1 \mu \mathrm{g} / \mathrm{ml}$ pepstatin A] for $4 \mathrm{~h}$ at $4^{\circ} \mathrm{C}$. After washing with buffer $\mathrm{A}$, the bound proteins were fractionated by SDS-PAGE and analyzed by Western blotting with antibodies against the largest subunit of RNA polymerase II (ARNA-3) (Progen) or MSL-1 antibody.

Yeast transactivation assay. The yeast strain Y190 was transformed with RHA deletion mutants using the lithium acetate method and transformants were selected on tryptophanfree agar plates for 3 days. For the liquid B-gal assay, $1 \mathrm{ml}$ of growth media was inoculated with selected colonies. The assay was performed in triplicate to quantify transcriptional activity as described previously (46).

Localization of GFP fusion proteins. SL-2 cells were transfected with $2 \mu \mathrm{g}$ of each construct using CellFectin reagent (Invitrogen). At $24 \mathrm{~h}$ after transfection, cells were seeded 


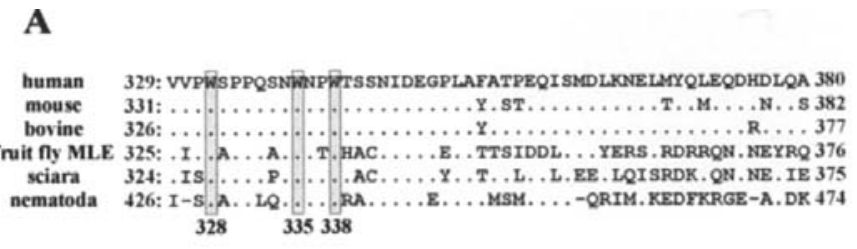

B
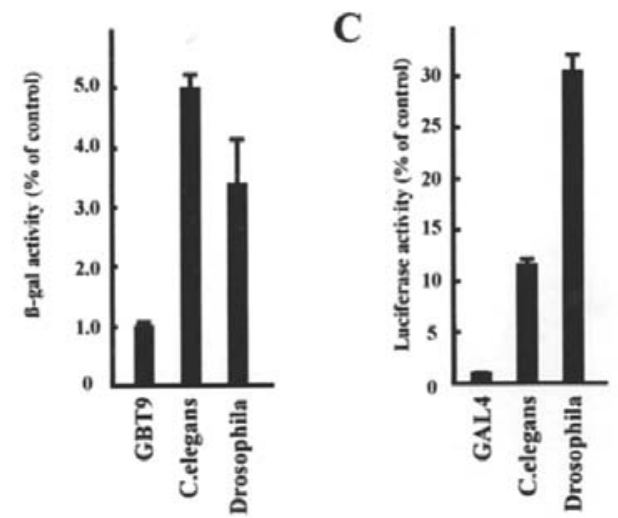

Figure 1. Transactivation through MTAD of MLE. (A) Alignment of MTAD sequences of RHA homologues from $C$. elegans to human. The MTAD sequences are well conserved among species. Transactivation assays with MTAD of C. elegans and Drosophila fused to GAL4-DBD in yeast (B) and HEK-293 cells (C). The relative B-gal or luciferase activity of each mutant was compared to that of the empty vector, which was designated as 1 .

onto 14- $\mathrm{mm}^{2}$ coverslips and incubated for $2 \mathrm{~h}$. Cells were washed 3 times with PBS and fixed with $3.7 \%$ formaldehyde and Tween-20 in PBS for $30 \mathrm{~min}$ at room temperature. After removal of formaldehyde, cells were washed with PBS. DNA was counterstained with DAPI for $10 \mathrm{~min}$ and washed with PBS and Tween-20, then the coverslips were mounted and analyzed by microscopy system (Leica).

Reporter assay. SL-2 cells $\left(1.2 \times 10^{5}\right)$ were plated in a $24-$ well plate. The cells were transiently transfected with $50 \mathrm{ng}$ of each mutant of MTAD fused to GAL4-DBD, or the empty vector alone, and $5 \mathrm{ng}$ of G14-ts/BasicVector2 using CellFectin. Following $30 \mathrm{~h}$ of incubation, cells were lysed with cell lysis buffer (Promega) and luciferase activities were measured. The measured activity was normalized for B-gal activity using cotransfected $B$-galactosidase derived by Actin$5 \mathrm{C}$ promoter as a control. Each experiment was performed in triplicate. For assay with the reporter plasmid of MLE target promoter, SL-2 cells were cotransfected with $10 \mathrm{ng}$ of DHSroX1, ProX2(-) or $1 \mathrm{ng}$ of heat shock reporter plasmid, and 25 or $50 \mathrm{ng}$ of MLE mutant expression vector. Cells were co-transfected with $25 \mathrm{ng}$ Actin5C $\beta$-gal as a control. The total amount of expression vector was kept constant by adding appropriate amounts of the empty vector pBlue-Actin5C.

\section{Results}

We reported previously that the transactivation domain of RHA consists of 50 amino acids termed MTAD. The MTAD interacts with Pol II complexes and activates CREBdependent transcription. The sequence of this domain is well conserved among RHA homologues from fly to human (Fig. 1A) (39).

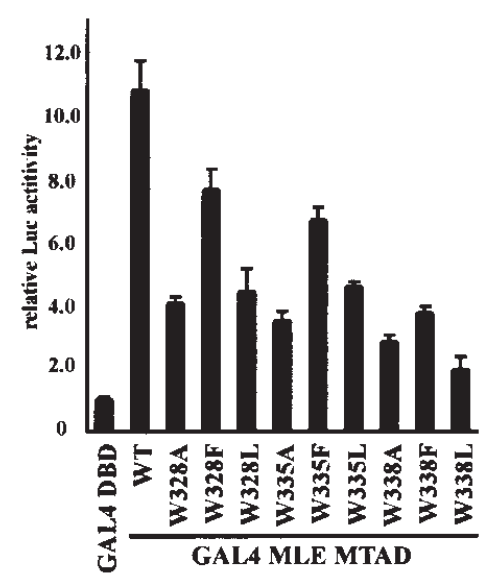

Figure 2. Transactivation assays in Drosophila cells with conserved residue mutants in MTAD. SL2 cells were transfected with $50 \mathrm{ng}$ of MTAD mutants fused to GAL4-DBD or empty vector, and $5 \mathrm{ng}$ of pG14-tp/BasicVector2 reporter plasmid. The relative luciferase activity derived from cells transformed with empty vector was designated as 1 . Each value of the relative luciferase activity represents the mean \pm SEM $(n=3)$.

We examined whether the functions of the MTAD as well as the amino acid sequences are conserved in the homologues. The transactivation assay with the MTAD of Caenorhobditis elegans RHA (426-474 aa) and Drosophila MLE (325-376 aa) fused to GAL4 DBD was performed in yeast and mammalian cells. In yeast, $C$. elegans MTAD and MLE MTAD were activated 5- or 3-fold more than GAL4 DBD alone, respectively, although their activities were lower than that of human RHA (Fig. 1B). Similar to the results in yeast, MTAD of C. elegans and MLE activated 12- or 13-fold more than empty vector, respectively (Fig. 1C). These results suggested that the function of MTAD as a transcriptional activator could be conserved beyond species.

To determine whether the MTAD functions in vivo as a transcriptional regulator beyond the species, we generated a transgenic fly with mle deleted MTAD ( $\mathrm{MTTAD})$ and performed complementation experiments. It has been reported that male homozygous for the spontaneous null mle mutant, named $\mathrm{mle}^{1}$, die at larval stage (27). The wild-type mle transgene fully complements the mutant phenotype (7), while the transgene lacking the ATP binding activity can not and the mutant die during the mid- to late-larval stage (26). We examined whether the transgene carrying deletion of that MTAD in mle was allowed to rescue the phenotype of null mutant. The 10.2-kb fragment containing the full-length mle gene and its flanking sequence was used as the transgene (7). The mle $\triangle$ MTAD transgene was deleted from the amino acid region 326 to 378 in the $10.2 \mathrm{~kb}$ fragment. The mle null mutant fly with the wild-type or mle $\triangle$ MTAD transgene were generated. As reported previously, the wild-type mle transgene rescued the lethality of mle null mutant, whereas the mle null mutant with mle $\triangle \mathrm{MTAD}$ transgene died during late larval stages similar to $\mathrm{mle}^{1}$ in the three independent lines. These results indicate that the MTAD region could be essential for the viability of mle flies.

Drosophila SL-2 cells derived from early embryo are commonly used as a model system for studying male functions. We examined the effects of MTAD on trans- 


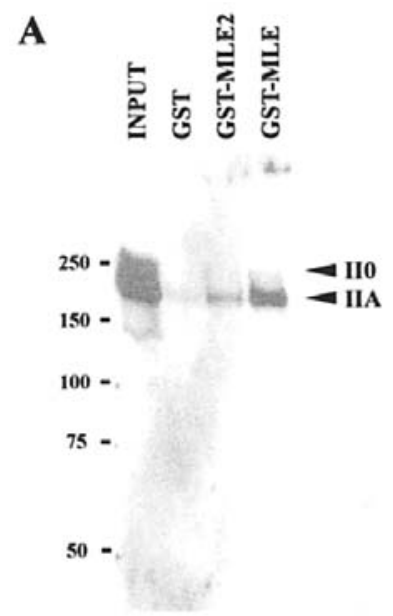

B



Figure 3. Interaction of MLE with Pol II in vitro. Nuclear extracts from SL2 cells were incubated with GST or MLE deletion mutant or full length fused to GST. Bound Pol II was visualized by Western blot analysis using an antibody against the largest subunit of Pol II.

activation using SL-2 cells. To confirm that MTAD has transcriptional activity in Drosophila cells, transactivation assay was performed in SL-2 cells. The wild-type MTAD of MLE fused with GAL4 DBD driven from Drosophila Actin5C promoter-activated transcription 11-fold more than an empty vector. We reported previously that three tryptophan residues at amino acids 332, 339 and 342 in MTAD of RHA are important for transactivation (47). To assess the functions of these conserved tryptophan residues in MLE, we fused a series of mutants with substitutions of tryptophan at amino acids 328, 335 and 338 with alanine, leucin and phenylalanine, with GAL4-DBD for transactivation assay. Mutants with substitutions of alanine or leucine exhibited only $35 \%$ of the transcriptional activity, compared with the wild-type MTAD. The mutants with substitution of phenylalanine retained higher activity, relative to that of mutants with substitution of other amino acids. Furthermore, substitutions of all tryptophan residues with alanines decreased the transcriptional activity to the same level as GAL4 DBD only (Fig. 2). These results suggest that the MTAD region of MLE also plays a role as a transactivation domain in SL-2 cells and that tryptophan residues are also important for the function of MTAD.

To test whether MLE recruits Pol II, a GST pull down assay was performed with MLE full-length and MLE2 region (219-653 aa) containing the MTAD region fused to GST. MLE bound with the hyperphosphorylation form of Pol II (Pol IIO) and the hypophosphorylation form of Pol II (Pol IIA) complexes, while GST alone did not (Fig. 3A). These results indicate that MLE interacted with both the elongation and initiation forms of Pol II. Furthermore, to confirm that the MTAD of MLE associates with Pol II complexes, we performed GST pull down assay with MLE2-deleted MTAD region (MLE2 $\triangle \mathrm{MTAD}$ ) and in which three tryptophan residues were substituted with alanines (MLE2AAA). Similar to RHA, MLE2 $\triangle$ MTAD and MLE2AAA did not interact with Pol II (Fig. 3B). These results indicate that MLE recruits Pol II through MTAD.

Then, we examined the localization of each mutant in cells, since deletion of a domain may affect the localization and functions. Each mutant of MLE was expressed as GFP fusion protein in SL-2 cells. The GFP was distributed throughout the whole cells, whereas the wild-type MLE accumulated also in some parts of the nucleus. It is known that mutants lacking the ATP binding ability colocalize with MSL-1 in immunostaining of the salivary gland (25). As reported previously, the mutant with substitution in the ATP binding site (MLEGET) was located in the nucleus similar to the wild-type. Surprisingly, MLE $\triangle$ MTAD showed both nuclear and cytoplasmic distribution, while MLEAAA was located in the nucleus similarly to the wild-type. We reported previously that RHA has the nuclear localization signal in the

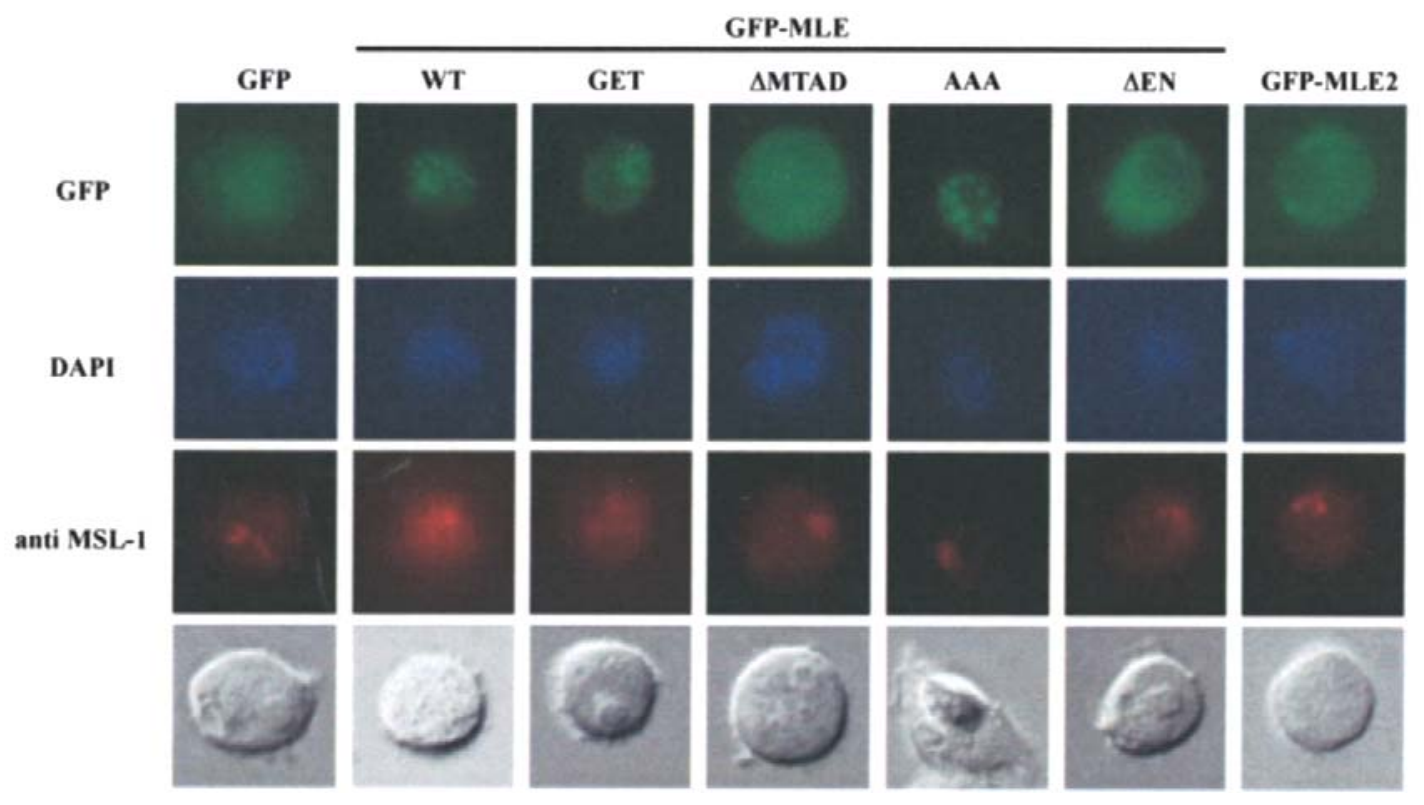

Figure 4. Localization of MLE mutants in SL-2 cells. SL-2 cells were transfected with the indicated MLE mutants fused to GFP. Localization of the fusion proteins was compared using immunofluorescence staining for GFP and MSL1, respectively. DNA was counterstained with DAPI. 
A

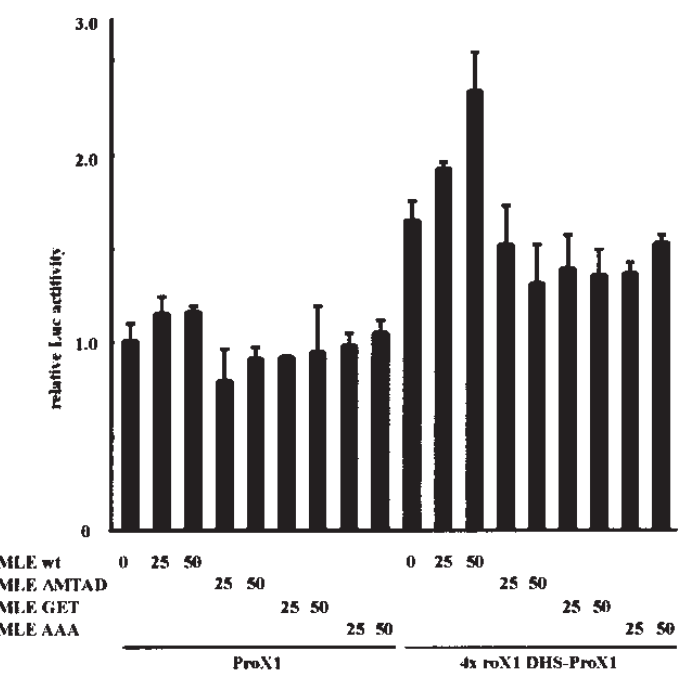

B

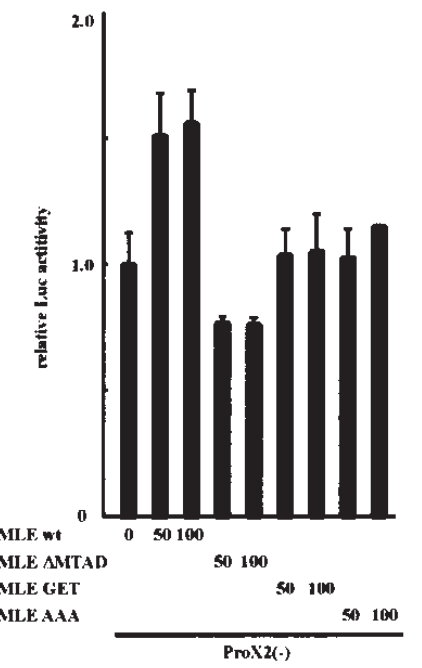

C

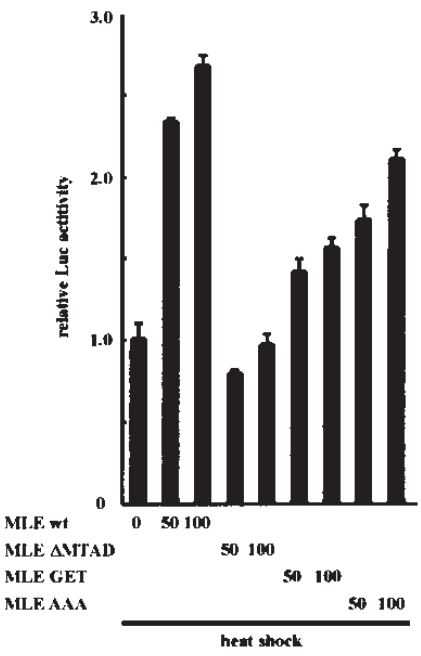

Figure 5. Effects of MTAD and tryptophan residue on MLE-dependent transcription. Transient reporter assays with roX1 (A), roX2 (B) and heat shock protein 70 promoter (C) Luc reporter. SL-2 cells were transfected with $10 \mathrm{ng}$ of $4 \mathrm{x}$ roX1 promoter-Luc, roX2 or $1 \mathrm{ng}$ hsp70 reporter plasmid, and indicated amount of each mutant of full-length MLE expression vector. The total amount of expression vector was kept constant with the addition of empty vector. Luciferase activity was normalized to $B$-gal. The relative luciferase activity of cells transfected with empty vector alone was designated as 1 . Each value of relative luciferase activity represents the mean $\pm \operatorname{SEM}(n=3)$.

C terminal region (41). To confirm whether the NLS of MLE locates in the MTAD, we assessed the localization of the deletion mutants including the MTAD (MLE2) and lacking the C-terminal region from 1163 to 1287 aa (MLE $\triangle \mathrm{EN})$. Both mutants were also localized in the cytoplasm similar to RHA (Fig. 4). These results indicated that MLE could have the NLS in its C-terminal region not in the MTAD, and showed that MTAD may play a role in intracellular distribution of MLE.

To confirm the contribution of MTAD to MLE-dependent gene expression, we examined the effects of the full-length MLE on the promoter of its target genes using the reporter assay. The DCC is known to activate the expression of roX1 and roX2 genes, which are one of the chromatin entry sites

A
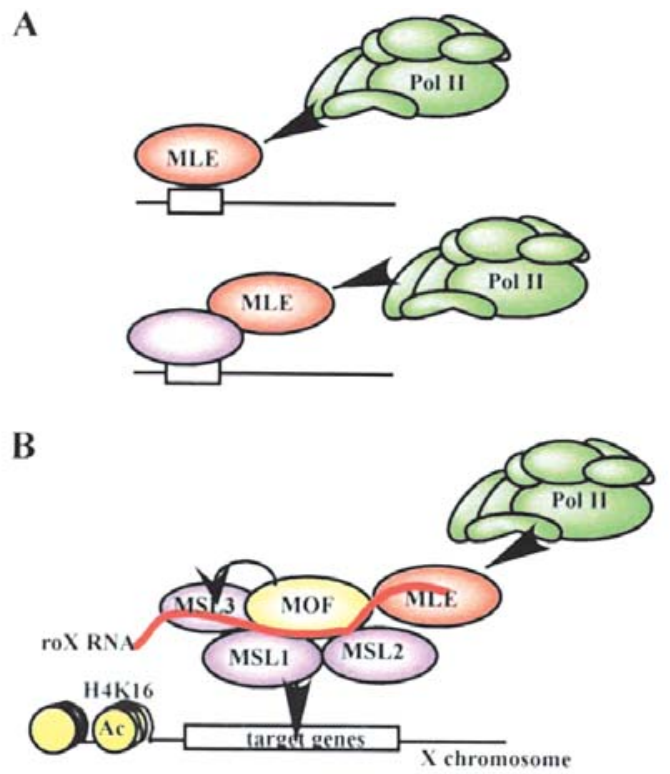

Figure 6. Schematic representations of transactivation mechanisms with MLE. (A) MLE may activate the transcriptional initiation through binding to promoters or transcription factors. (B) MLE works as a component of the $\mathrm{DCC}$ and was able to regulate the gene expression from $\mathrm{X}$ chromosome.

on $\mathrm{X}$ chromosome, through binding to the DHS in those genes $(18,21)$. We utilized the promoter region of roX1 fused to DHS, which locates at the center of roX1 gene (18) as a DCC target gene. The roX1 promoter (ProX1) with the DHS was activated 2 -fold higher than with ProX1 only. Thus, MLE had only slight effect on the transactivation derived from roX1 promoter (ProX1), while MLE activated the DHS dependent transcription 1.5 -fold higher than the empty vector (Fig. 5A).

MLE belongs to an ATPase/helicase family and contains conserved ATP binding motifs. The enzymatic activity of MLE is essential for its functions in vivo (26). As expected, MLE mutant lacking the ATP binding ability (MLEGET) could not activate. The mutants lacking the Pol II binding ability (MLEAMTAD and MLEAAA) activated to the same levels as the empty vector. Then, we examined the effects of MTAD on transcription of MLE-regulated promoters. It was reported that MLE is recruited to the promoter of roX2 gene. The roX2 gene contains the MLE binding site to which MSL-1 does not interact in addition to the DHS (45). Moreover, a previous study indicated that MLE localizes on the heat shock gene cluster of chromosome 3 , which show a localization pattern independent of the DCC (48). In this assay, we used the hypothetical promoter region (ProX2) of the roX2 gene at -207 to 92 lacking the DHS [ProX2(-)] (45) and the heat shock promoter (hsp70) as the reporter. The wild-type MLE-activated ProX2 and heat shock promoters by 1.5 - or 2 -fold compared to empty vector, respectively. The activities derived from MLEGET and MLEAAA were reduced to $50 \%$ of the levels induced by wild-type MLE. The activity of MLE $\triangle M T A D$ was lower than the empty vector (Fig. 5B and C). These results suggest that MTAD, especially tryptophan residues, are important for transactivation of MLE target genes. 


\section{Discussion}

In this study, we confirmed the functions of Drosophila MTAD of MLE on transactivation in fruit fly cells. In vivo, the wild-type MLE complemented the spontaneous MLE null mutant, while MLEAMTAD failed to rescue the lethality. In SL-2 cells, this domain exhibited transcriptional activity (Fig. 2) and ability for recruitment of Pol II (Fig. 3). MLE regulated the transactivation of the target genes through MTAD (Fig. 5).

The DCC is sequentially formed and recruited to the target genes on $\mathrm{X}$ chromosome. After activating the expression of the target genes driven from the high affinity entry site, the DCC spreads onto the low affinity sites of the X chromosome (19). There is much progress in our understanding of the interactions, functions of MSLs and roX RNAs and mechanisms of each step in dosage compensation. MLE seems essential for the survival of male fly and its enzymatic activities are necessary for its roles $(26,27)$. MLE, MOF and MSL-3 cooperate with each other for the formation of the DCC and localization on the X chromosome (24). In addition to the in vivo data, it was reported that MLE is recruited to the roX2 gene promoter and activates the transcription $(21,45)$. However, there is little information on the functions of MLE in the DCC on the tansactivation step, including initiation and elongation, or the spreading step in the dosage compensation. In the present study, MTAD was confirmed to have transcriptional activity (Fig. 2) and the results indicated that three tryptophan residues in MTAD are important for association with Pol II (Fig. 3). These results suggest that MLE acted as a recruiting factor for the general transcriptional machinery to the DCC or X chromosome. It is also reported that MSL-2 fused to GAL4-DBD has transcriptional activity (49). These findings allow us to speculate on some possibilities in transactivation mechanism through the DCC. MLE and MSL-2 may act as activators synergistically or MSL-2 as a scaffold factor and recruit MLE. The mechanisms of recruitment of Pol II may be different between the core complex, which comprises of only MSL-1 and MSL-2, and the mature DCC that includes all components. In addition, the roles of ATPase and helicase activity in the dosage compensation remain poorly understood. More detailed analysis is needed to understand the transactivation mechanisms.

In this study, we confirmed the distribution of each MLE mutant in SL-2 cells. In Fig. 4, the wild-type MLE was detected in the nucleus. The mutant with deleted MTAD was defused throughout the whole cells. In our previous study, we indicated the effects of various functional domains in RHA on the nuclear localization. The wild-type RHA was located in the nucleus, while RHAW339A lacking the Pol II binding ability was partially localized in the cytoplasm. These results suggest that the MTAD may contribute to the retention in the nucleus (50). Furthermore, the Pol II binding ability may be important for the localization in the nucleus. Since the NLS of MLE is located in the C-terminus, similar to RHA (Fig. 4), it is thought that MLE can be imported into the nucleus but not retained there. It was reported that deletion of roX1 and roX2 genes lead to mis-location of the DCC in cells (51). It has also been speculated that the MTAD could work as a nuclear retention signal, from the mature DCC on X chromosome or have unknown functions in addition to transactivation in cells.

Several studies have emphasized the multi-functional roles of MLE in gene expression as well as gene dosage compensation in male flies. Recently, studies using immunostaining and ChIP assay revealed that the localization of components were related to the dosage compensation on chromosomes (52-55). Immunostaining of polytene chromosome showed the DCC colocalizes with a subset of Pol IIO on the $\mathrm{X}$ chromosome in male flies, although the DCC does not associate with all transcriptional active sites (48). Acetylation of $\mathrm{K} 16$ in histone $\mathrm{H} 4$ is found in the coding regions and $3^{\prime}$ end of $\mathrm{X}$-chromosomal genes (52). However, there is no information on the localization of Pol IIA on X chromosome, due to the use of anti-Pol II antibody against all forms or the elongation form. Moreover, the majority of MSL-1 binding sites on the $\mathrm{X}$ chromosome are not consistent with the Pol II binding sites (52). Taken together, we consider that the DCC is rather important for the spreading of the DCC and elongation than initiation. In our study, MLE interacted also with Pol IIO but mainly with Pol IIA. It is known that some factors, such as LMP2 (low molecular mass polypeptide 2), function not only during transcription initiation but also during transcription elongation (56). We speculate that MLE included in the DCC could associate with the Pol IIO and regulates elongation, whereas the single MLE not as a complex may recruit Pol IIA to the promoters and activate the transcription (Fig. 6). Future studies should determine the steps at which each component of the DCC works.

In mammalian cells, RHA has certain functions in gene expression. Our study showed that RHA interacts with various transcriptional activators including CBP. It acts as a coactivator recruiting general transcriptional factors such as Pol II and mediates transactivation $(34,39)$. In the present study, the results suggest that Drosophila homologue MLE activated transcription, through Pol II to the target genes similar to RHA. MLE is also suggested to have additional functions independent of the DCC in transactivation. MLE activated the transcription derived from the heat shock promoter, whereas other components of the DCC, msl-1, msl-2 and msl-3, had only slight effects (Fig. 4). These data are consistent with previous reports (48). MLE is found in puffs in which the DCC does not localize. In female, MLE is detected on some sites more strongly than in male. Furthermore, it localizes in the puffs of autosomal chromosomes as well as on $\mathrm{X}$ chromosome. These puffs include a cluster of hsp70 genes (48). These results suggest that MLE could work in trans-activation alone not as a component of the DCC. In mammalian cells, RHA is known to activate transcription through binding to the promoter as well as binding with transcription factors $(33,57)$. It was reported that MLE promotes expression of roX2 gene through the element that does not include DHS on ProX2 promoter (45). These data suggest that MLE, unlike the DCC, could have transactivation functions. RHA has many roles not only in transcription but also in transportation of viral RNAs and translation (33,58-60). In addition, a new function of RHA was identified recently; RHA is a component of RISC (RNAinduced silencing complex) and promotes the assembly of 
the complex (61). The functional domains of MLE are well conserved between RHA and MLE (39). These findings allow us to speculate that various functions of RHA are also conserved in MLE. Exploring the associating factors with MLE should enhance our understanding of the transcriptional mechanisms, furthermore to understand the regulation mechanisms of gene expression in eukaryotic cells.

\section{Acknowledgements}

We thank Dr Yasushi Hiromi for the plasmid pWA GAL4. We also thank Sanae Shinkawa, Yukari Nakagawa and Mayumi Hanaoka for technical assistance. This work was partially supported financially by the Japanese Ministry of Education, Science Culture and Sports; the Japanese Ministry of Health and Welfare, Japan Science and Technology Corporation; the Human Health Science Foundation; Kanagawa Academy of Science and Technology Research Grants, the Kanae Foundation for Life and Socio-Medical Science; the Kanagawa Nanbyo Foundation; the Kanagawa High-Technology Foundation; Kanagawa Academy of Science and Technology Research Grants; Ministry of Education, Culture, Sports and Technology; Japan Society for Promotion of Science, and Ministry of Health, Labour and Welfare.

\section{References}

1. Mukherjee AS and Beermann W: Synthesis of ribonucleic acid by the X-chromosomes of Drosophila melanogaster and the problem of dosage compensation. Nature 207: 785-786, 1965.

2. Palmer MJ, Mergner VA, Richman R, Manning JE, Kuroda MI and Lucchesi JC: The male-specific lethal-one (msl-1) gene of Drosophila melanogaster encodes a novel protein that associates with the $X$ chromosome in males. Genetics 134: 545-557, 1993.

3. Palmer MJ, Richman R, Richter L and Kuroda MI: Sex-specific regulation of the male-specific lethal-1 dosage compensation gene in Drosophila. Genes Dev 8: 698-706, 1994.

4. Bashaw GJ and Baker BS: The msl-2 dosage compensation gene of Drosophila encodes a putative DNA-binding protein whose expression is sex specifically regulated by Sex-lethal. Development 121: 3245-3258, 1995.

5. Gorman M, Franke A and Baker BS: Molecular characterization of the male-specific lethal-3 gene and investigations of the regulation of dosage compensation in Drosophila. Development 121: 463-475, 1995 .

6. Hilfiker A, Hilfiker-Kleiner D, Pannuti A and Lucchesi JC: mof, a putative acetyl transferase gene related to the Tip60 and MOZ human genes and to the SAS genes of yeast, is required for dosage compensation in Drosophila. EMBO J 16: 2054-2060, 1997.

7. Kuroda MI, Kernan MJ, Kreber R, Ganetzky B and Baker BS: The maleless protein associates with the $\mathrm{X}$ chromosome to regulate dosage compensation in Drosophila. Cell 66: 935-947, 1991.

8. Kelley RL, Solovyeva I, Lyman LM, Richman R, Solovyev V and Kuroda MI: Expression of msl-2 causes assembly of dosage compensation regulators on the $\mathrm{X}$ chromosomes and female lethality in Drosophila. Cell 81: 867-877, 1995.

9. Uenoyama T, Fukunaga A and Ioshi K: Studies on the sex-specific lethals of Drosophila melanogaster. V. Sex transformation caused by interactions between a female-specific lethal, Sxlf 1, and the male-specific lethals mle(3)132, msl-2(27), and mle. Genetics 102: 233-243, 1982.

10. Meller VH, Wu KH, Roman G, Kuroda MI and Davis RL: roX1 RNA paints the X chromosome of male Drosophila and is regulated by the dosage compensation system. Cell 88: 445-457, 1997.

11. Amrein H and Axel R: Genes expressed in neurons of adult male Drosophila. Cell 88: 459-469, 1997.

12. Park Y, Kelley RL, Oh H, Kuroda MI and Meller VH: Extent of chromatin spreading determined by roX RNA recruitment of MSL proteins. Science 298: 1620-1623, 2002.
13. Bone JR, Lavender J, Richman R, Palmer MJ, Turner BM and Kuroda MI: Acetylated histone H4 on the male X chromosome is associated with dosage compensation in Drosophila. Genes Dev 8: 96-104, 1994.

14. Turner BM, Birley AJ and Lavender J: Histone H4 isoforms acetylated at specific lysine residues define individual chromosomes and chromatin domains in Drosophila polytene nuclei. Cell 69: 375-384, 1992.

15. Mendjan S and Akhtar A: The right dose for every sex. Chromosoma 116: 95-106, 2007.

16. Akhtar A: Dosage compensation: an intertwined world of RNA and chromatin remodelling. Curr Opin Genet Dev 13: 161-169, 2003.

17. Kelley RL, Meller VH, Gordadze PR, Roman G, Davis RL and Kuroda MI: Epigenetic spreading of the Drosophila dosage compensation complex from roX RNA genes into flanking chromatin. Cell 98: 513-522, 1999.

18. Kageyama Y, Mengus G, Gilfillan G, et al: Association and spreading of the Drosophila dosage compensation complex from a discrete roX1 chromatin entry site. EMBO J 20: 2236-2245, 2001 .

19. Gilfillan GD, Dahlsveen IK and Becker PB: Lifting a chromosome: dosage compensation in Drosophila melanogaster. FEBS Lett 567: 8-14, 2004.

20. Akhtar A and Becker PB: Activation of transcription through histone $\mathrm{H} 4$ acetylation by MOF, an acetyltransferase essential for dosage compensation in Drosophila. Mol Cell 5: 367-375, 2000.

21. Bai X, Alekseyenko AA and Kuroda MI: Sequence-specific targeting of MSL complex regulates transcription of the roX RNA genes. EMBO J 23: 2853-2861, 2004.

22. Park Y, Mengus G, Bai X, et al: Sequence-specific targeting of Drosophila roX genes by the MSL dosage compensation complex. Mol Cell 11: 977-986, 2003.

23. Meller VH, Gordadze PR, Park Y, et al: Ordered assembly of roX RNAs into MSL complexes on the dosage-compensated X chromosome in Drosophila. Curr Biol 10: 136-143, 2000.

24. Scott MJ, Pan LL, Cleland SB, Knox AL and Heinrich J: MSL1 plays a central role in assembly of the MSL complex, essential for dosage compensation in Drosophila. EMBO J 19: 144-155, 2000.

25. Richter L, Bone JR and Kuroda MI: RNA-dependent association of the Drosophila maleless protein with the male $\mathrm{X}$ chromosome. Genes Cells 1: 325-336, 1996.

26. Lee CG, Chang KA, Kuroda MI and Hurwitz J: The NTPase/ helicase activities of Drosophila maleless, an essential factor in dosage compensation. EMBO J 16: 2671-2681, 1997.

27. Fukunaga A, Tanaka A and Oishi K: Maleless, a recessive autosomal mutant of Drosophila melanogaster that specifically kills male zygotes. Genetics 81: 135-141, 1975.

28. Pannuti A and Lucchesi JC: Recycling to remodel: evolution of dosage-compensation complexes. Curr Opin Genet Dev 10: 644-650, 2000.

29. Marin I: Evolution of chromatin-remodeling complexes: comparative genomics reveals the ancient origin of 'novel' compensasome genes. J Mol Evol 56: 527-539, 2003

30. Smith ER, Cayrou C, Huang R, Lane WS, Cote J and Lucchesi JC: A human protein complex homologous to the Drosophila MSL complex is responsible for the majority of histone H4 acetylation at lysine 16. Mol Cell Biol 25: 9175-9188, 2005.

31. Lee CG and Hurwitz J: Human RNA helicase A is homologous to the maleless protein of Drosophila. J Biol Chem 268: 16822-16830, 1993.

32. Fujita H, Fujii R, Aratani S, Amano T, Fukamizu A and Nakajima T: Antithetic effects of MBD2a on gene regulation. Mol Cell Biol 23: 2645-2657, 2003.

33. Fujii R, Okamoto M, Aratani S, et al: A role of RNA helicase A in cis-acting transactivation response element-mediated transcriptional regulation of human immunodeficiency virus type 1. J Biol Chem 276: 5445-5451, 2001.

34. Nakajima T, Uchida C, Anderson SF, et al: RNA helicase A mediates association of CBP with RNA polymerase II. Cell 90: 1107-1112, 1997.

35. Zhang SS and Grosse F: Purification and characterization of two DNA helicases from calf thymus nuclei. J Biol Chem 266: 20483-20490, 1991.

36. Lee CG and Hurwitz J: A new RNA helicase isolated from HeLa cells that catalytically translocates in the $3^{\prime}$ to $5^{\prime}$ direction. J Biol Chem 267: 4398-4407, 1992. 
37. Zhang S and Grosse F: Nuclear DNA helicase II unwinds both DNA and RNA. Biochemistry 33: 3906-3912, 1994.

38. Zhang S and Grosse F: Domain structure of human nuclear DNA helicase II (RNA helicase A). J Biol Chem 272: 11487-11494, 1997.

39. Aratani S, Fujii R, Oishi T, et al: Dual roles of RNA helicase A in CREB-dependent transcription. Mol Cell Biol 21: 4460-4469, 2001.

40. Tang H, McDonald D, Middlesworth T, Hope TJ and Wong-Staal F: The carboxyl terminus of RNA helicase A contains a bidirectional nuclear transport domain. Mol Cell Biol 19: 3540-3550, 1999.

41. Aratani S, Oishi T, Fujita H, et al: The nuclear import of RNA helicase $\mathrm{A}$ is mediated by importin-alpha3. Biochem Biophys Res Commun 340: 125-133, 2006.

42. Anderson SF, Schlegel BP, Nakajima T, Wolpin ES and Parvin JD: BRCA1 protein is linked to the RNA polymerase II holoenzyme complex via RNA helicase A. Nat Genet 19: 254-256, 1998

43. Kitagawa H, Yanagisawa J, Fuse H, et al: Ligand-selective potentiation of rat mineralocorticoid receptor activation function 1 by a CBP-containing histone acetyltransferase complex. Mol Cell Biol 22: 3698-3706, 2002.

44. Myohanen S and Baylin SB: Sequence-specific DNA binding activity of RNA helicase A to the p16INK4a promoter. J Biol Chem 276: 1634-1642, 2001.

45. Lee CG, Reichman TW, Baik T and Mathews MB: MLE functions as a transcriptional regulator of the roX2 gene. J Biol Chem 279: 47740-47745, 2004.

46. Yoshida E, Aratani S, Itou H, et al: Functional association between CBP and HNF4 in trans-activation. Biochem Biophys Res Commun 241: 664-669, 1997.

47. Aratani S, Fujii R, Fujita H, Fukamizu A and Nakajima T: Aromatic residues are required for RNA helicase A mediated transactivation. Int J Mol Med 12: 175-180, 2003.

48. Kotlikova IV, Demakova OV, Semeshin VF, et al: The Drosophila dosage compensation complex binds to polytene chromosomes independently of developmental changes in transcription. Genetics 172: 963-974, 2006.

49. Straub T, Neumann MF, Prestel M, et al: Stable chromosomal association of MSL2 defines a dosage-compensated nuclear compartment. Chromosoma 114: 352-364, 2005.
50. Fujita H, Ohshima T, Oishi T, et al: Relevance of nuclear localization and functions of RNA helicase A. Int J Mol Med 15: 555-560, 2005.

51. Meller VH and Rattner BP: The roX genes encode redundant male-specific lethal transcripts required for targeting of the MSL complex. EMBO J 21: 1084-1091, 2002.

52. Gilfillan GD, Straub T, de Wit E, et al: Chromosome-wide gene-specific targeting of the Drosophila dosage compensation complex. Genes Dev 20: 858-870, 2006.

53. Schubeler D: Dosage compensation in high resolution: global up-regulation through local recruitment. Genes Dev 20: 749-753, 2006.

54. Alekseyenko AA, Larschan E, Lai WR, Park PJ and Kuroda MI: High-resolution ChIP-chip analysis reveals that the Drosophila MSL complex selectively identifies active genes on the male $\mathrm{X}$ chromosome. Genes Dev 20: 848-857, 2006.

55. Legube G, McWeeney SK, Lercher MJ and Akhtar A: Xchromosome-wide profiling of MSL-1 distribution and dosage compensation in Drosophila. Genes Dev 20: 871-883, 2006.

56. Zhang H, Sun L, Liang J, et al: The catalytic subunit of the proteasome is engaged in the entire process of estrogen receptorregulated transcription. EMBO J 25: 4223-4233, 2006.

57. Zhong $X$ and Safa AR: RNA helicase $A$ in the MEF1 transcription factor complex up-regulates the MDR1 gene in multidrug-resistant cancer cells. J Biol Chem 279: 17134-17141, 2004.

58. Tang $\mathrm{H}$ and Wong-Staal F: Specific interaction between RNA helicase A and Tap, two cellular proteins that bind to the constitutive transport element of type D retrovirus. J Biol Chem 275: 32694-32700, 2000.

59. Li J, Tang H, Mullen TM, et al: A role for RNA helicase A in post-transcriptional regulation of HIV type 1. Proc Natl Acad Sci USA 96: 709-714, 1999.

60. Yang JP, Tang H, Reddy TR and Wong-Staal F: Mapping the functional domains of HAP95, a protein that binds RNA helicase A and activates the constitutive transport element of type D retroviruses. J Biol Chem 276: 30694-30700, 2001.

61. Robb GB and Rana TM: RNA helicase A interacts with RISC in human cells and functions in RISC loading. Mol Cell 26: 523-537, 2007. 